\title{
Identification of key pathways and genes in vestibular schwannoma using bioinformatics analysis
}

\author{
BO WU ${ }^{1,2^{*}}$, GAOJING DOU ${ }^{1,3^{*}}$, YUAN ZHANG $^{1}$, JING WANG $^{1}$, XINHUI WANG ${ }^{1,4}$, \\ SHANSHAN JIANG ${ }^{5}$, SHENG ZHONG $^{6}$, JUNAN REN ${ }^{1}$, ZHIYUN ZHANG $^{1}$, \\ JIAHUI LI ${ }^{7}$, CHUNJIA SHENG $^{1}$, GANG ZHAO $^{8}$ and LIYAN ZHAO ${ }^{9}$ \\ ${ }^{1}$ Clinical College, Jilin University; Departments of ${ }^{2}$ Orthopedics, ${ }^{3}$ Breast Surgery and ${ }^{4}$ Oncology, \\ The First Bethune Hospital of Jilin University, Changchun, Jilin 130021; ${ }^{5}$ Institute of Zoology, China Academy of Science, \\ Beijing 100049; ${ }^{6}$ Department of Neurosurgery, Cancer Hospital of Sun Yat Sen University, \\ Guangzhou, Guangdong 510060; ${ }^{7}$ Department of Pharmacy, West China Hospital, Sichuan University, Chengdu, \\ Sichuan 610041; ${ }^{8}$ Department of Neurosurgery, The First Bethune Hospital of Jilin University, Changchun, Jilin 130021; \\ ${ }^{9}$ Department of Clinical Laboratory, The Second Hospital of Jilin University, Changchun, Jilin 130041, P.R. China
}

Received April 9, 2020; Accepted October 6, 2020

DOI: $10.3892 /$ etm.2022.11141

\begin{abstract}
The aim of the present study is to identify novel promising marks and targets of diagnosis, therapy and prognosis for patients with vestibular schwannoma at the molecular level. The gene expression profiles of GSE54934, GSE39645 and GSE56597 datasets were obtained respectively from the Gene Expression Omnibus database. The differentially expressed genes (DEGs) were identified by comparing between gene expression profiles of the vestibular schwannoma tissues and normal tissues. Subsequently, Gene Ontology (GO), Kyoto Encyclopedia of Genes and Genomes (KEGG) analysis and protein-protein interaction (PPI) network analysis were performed. The function and pathway enrichment analysis were performed for DEGs with DAVID. Reverse transcription-quantitative PCR were conducted to confirm the expression of BCL2, AGT, IL6 and ITGA2 in human Schwann cells and vestibular schwannoma cells. A total of 4,025, 1,1291 and 1,513 DEGs were identified from GSE54934, GSE56597 and GSE39645 datasets, respectively. GO and KEGG analysis showed that the mutual upregulated genes were mainly enriched in cell division, mitotic nuclear division, and transition of mitotic cell cycle, whilst mutual downregulated genes were enriched in chemical synaptic transmission, neurotransmitter transport, and synaptic vesicle membrane. Subsequently,
\end{abstract}

Correspondence to: Professor Liyan Zhao, Department of Clinical Laboratory, The Second Hospital of Jilin University, 218 Ziqiang Street, Changchun, Jilin 130041, P.R. China

E-mail: zhaoliy@jlu.edu.cn

${ }^{*}$ Contributed equally

Key words: bioinformatics, brain science, diagnosis, vestibular schwannoma
20 genes, including BCL2, AGT, IL6 and ITGA2 were selected as hub genes with high degrees after PPI network analysis. The significant differential expression of those genes were detected among vestibular schwannoma tissues compared with normal nerve tissues. In conclusion, BCL2, AGT, IL6 and ITGA2 are significantly higher expressed in vestibular schwannoma tissues compared with human Schwann tissues. The DEGs identified in the present study provide novel targets for the diagnosis and treatment of vestibular schwannoma.

\section{Introduction}

Vestibular schwannoma (VS), also known as vestibular Schwann cell tumor or acoustic neuroma (AN), is a type of primary tumor in the nerve sheath of the junction between the cranial nerve VIII vestibular branch center and peripheral nerves (1). VS is a common intracranial tumor, accounting for $\sim 90 \%$ of cerebellopontine angle tumors (2).

The vast majority of VS are benign tumors, while malignant tumors or malignant transformation are rarely seen. The type of VS varies, but in most cases the tumor grows slowly at an annual rate of $\sim 1-2 \mathrm{~mm}$ (3). Furthermore, a part of the tumor would stop growing when it reaches a certain size, or undergoes auto-atrophy and become smaller (3). However, large tumors may still remain in the brain stem and cause compression or intracranial hypertension, which threaten patient's life when the symptoms are severe. VS is mostly disseminated unilateral lesion, though some are family genetic bilateral lesions. For different sizes and types of the VS, the current treatment methods include surgical resection, stereotactic radiotherapy and long-term follow-up observation (4). Nevertheless, patients are always at risk of serious intracranial complications after surgery or radiotherapy. Accordingly, such treatment outcome effect is unsatisfactory for both patients and doctors. Thus, there is an urgent need for a comprehensive and specific method to reduce the risk of postoperative complications and to improve the situation. 
In recent years, accumulating evidence has suggested that various types of genes and cellular pathways contribute to the occurrence and development of VS. A study by Martini et al (5), which was conducted on YAP, TAZ and AREG expression in eighth cranial nerve schwannoma, proved that there was a significant direct correlation between TAZ expression and vestibular schwannoma volumes. In addition, Zhang et al (6) reported on a cDNA microarray analysis on VS and solid vestibular schwannoma models, which demonstrates a mutation spectrum and differential gene expression in cystic and solid vestibular schwannoma, which could provide useful insights into the molecular mechanism. However, few current studies had expounded a comprehensive and precise molecular mechanism related to VS progression and pathogenesis; nevertheless, the current understanding of VS is still limited, superficial and provincial. Therefore, there is a need for comprehensive research to improve the present situation.

Microarray technology combined with bioinformatics made it possible to analyze the genetic alteration and molecular mechanisms in the development and progression of VS, which may provide a theoretical basis for target prevention in VS. In the present study, gene expression profiles of VS tissues and the normal tissues were analyzed to identify the discrepancy, in order to define differentially expressed genes (DEGs). Subsequently, Gene Ontology (GO), Kyoto Encyclopedia of Genes and Genomes (KEGG) pathway enrichment analysis and protein-protein interaction (PPI) network analysis were conducted, as well as reverse transcription-quantitative (RT-q) PCR analysis. The aim of the present study was to provide data to determine the comprehensive and profound mechanism of VS, biomarkers for diagnosis and prognosis of VS, and novel targets for treatment of patients with VS.

\section{Materials and methods}

Microarray data. The expression profiles of GSE54934 (https:// www.ncbi.nlm.nih.gov/geo/query/acc.cgi?acc=GSE54934), GSE39645 (https://www.ncbi.nlm.nih.gov/geo/query/acc. cgi?acc=GSE39645) and GSE56597 (https://www.ncbi.nlm. nih.gov/geo/query/acc.cgi?acc=GSE56597) were obtained from Gene Expression Omnibus (GEO, http://www.ncbi.nlm. nih.gov/geo) database of National Center of Biotechnology Information (NCBI) (http://www.ncbi.nlm.nih.gov/). The array data of GSE54934 contained 31 VS samples and 9 normal nerve samples. GSE39645 includes 31 VS samples and 9 normal nerve samples. GSE56597 contains 31 VS samples and 9 normal nerve samples (7-9).

Data preprocessing and identification of DEGs. After obtaining the data, the GeneSpring software (version 11.5; Agilent Technologies, Inc.) was used to analyze the DEGs that were identified. The data of every series was categorized into two groups (VS and normal). Hierarchical clustering analysis and principle component analysis (PCA) were applied to determined probe quality control in GeneSpring. Probes, where intensity values below 20th percentile were filtered out with 'Filter ProbeSets by Expression' option. Within series, unpaired t-test was used to identify DEGs with a change $>2.0$ fold and defined a P-value cutoff of $<0.05$ to be considered as statistically significant. Then, Venn plot and analysis were conducted using website tools (http://bioinformatics.psb.ugent. be/webtools/Venn/).

Functional and pathway enrichment analysis. The Database for Annotation, Visualization and Integrated Discovery (DAVID; http://david.abcc.ncifcrf.gov/) is an online program applied to provide a comprehensive set of functional annotation tools to perceive biological meaning underlying a group of genes. GO (http://www.geneontology.org/) enrichment analysis is a method to analyze biological process, molecular function and cell component. KEGG pathway enrichment analysis was used to identify DEGs using the DAVID database. $\mathrm{P}<0.05$ was considered to indicate a statistically significant difference.

PPI network and module analysis. Search Tools for Retrieval of Interacting Genes (STRING; http://string.embl.de/) database and Cytoscape (v 3.8.2) software (The Cytoscape Consortium) were used to construct the protein-protein interaction (PPI) network of DEGs $(10,11)$. Subsequently, the Molecular Complex Detection (MCODE) was performed to screen hub genes and modules. $\mathrm{P}<0.05$ was considered to indicate a statistically significant difference. Subsequently, the function and pathway enrichment analysis were performed for DEGs with DAVID.

Cell lines. XY-H350 (human Schwann cell line), HEI193 cell line and RT4-D6P2T cell line (schwannoma cell line) were obtained from the American Type Culture Collection. All cell lines were cultured in Dulbecco's modified Eagle's medium (HyClone; Cytvia) supplemented with $10 \%$ fetal bovine serum (Gibco; Thermo Fisher Scientific, Inc.). An atmosphere of $5 \% \mathrm{CO}_{2}$ and $95 \%$ air at $37^{\circ} \mathrm{C}$ was maintained for the cultivation of the cell lines.

$R T-q P C R$. In order to confirm the expression of BCL2, AGT, IL6 and ITGA2 in the human Schwann cell line and schwannoma cell lines, RT-qPCR was performed in triplicate in a CFX96 Real-Time System (Bio-Rad Laboratories, Inc.) with a FastStart Universal SYBR Green Master (ROX) (Roche Diagnostics) according to the manufacturer's instructions and expression levels were normalized to glyceraldehyde-3-phosphate dehydrogenase (GAPDH). TRIzol ${ }^{\circledR}$ reagent (Thermo Fisher Scientific, Inc.) was utilized to isolate RNA from tissues and cells, as per the guidelines provided by the manufacturer. The PrimeScript RT-qPCR kit (Takara Bio, Inc.) was utilized for reverse transcription to obtain cDNA. To quantify gene expression, two-Step RT-qPCR was performed with hot start Taq at $95^{\circ} \mathrm{C}(15 \mathrm{sec})$, with annealing and extension at $60^{\circ} \mathrm{C}(60 \mathrm{sec})$ for 40 cycles, followed by a melting curve analysis. All RT-qPCR data were analyzed using the $2^{-\Delta \Delta C q}$ method (12). Primers (Shanghai GenePharma Co., Ltd.) were designed through Primer Bank (https://pga.mgh.harvard. edu/primerbank/) and were re-checked by NCBI Primer-Blast (http://www.ncbi.nlm.nih.gov/tools/primer-blast/). The following gene-specific primers were used: BCL2 sense, 5'-GGTGGGGTCATGTGTGTGG-3'; BCL2 anti-sense, 5'-CGGTTCAGGTACTCAGTCATCC-3'; AGT sense, CCC CAGTCTGAGATGGCTC; AGT anti-sense, GACGAGGTG GAAGGGGTGTA; IL6 sense, ACTCACCTCTTCAGAACG 
A
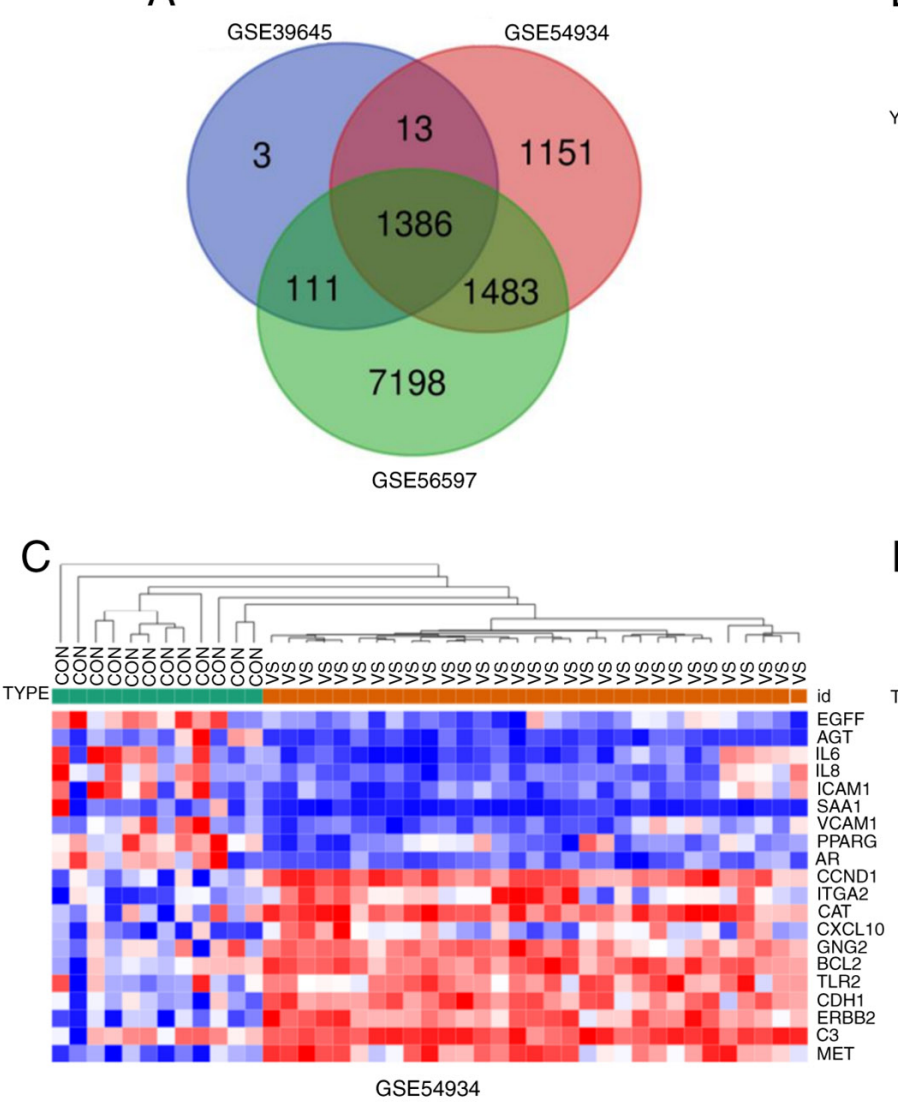

B

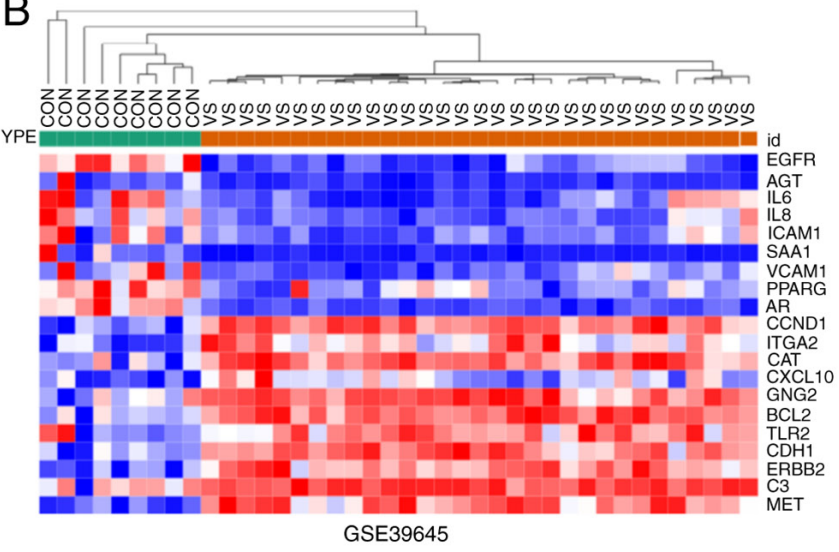

D

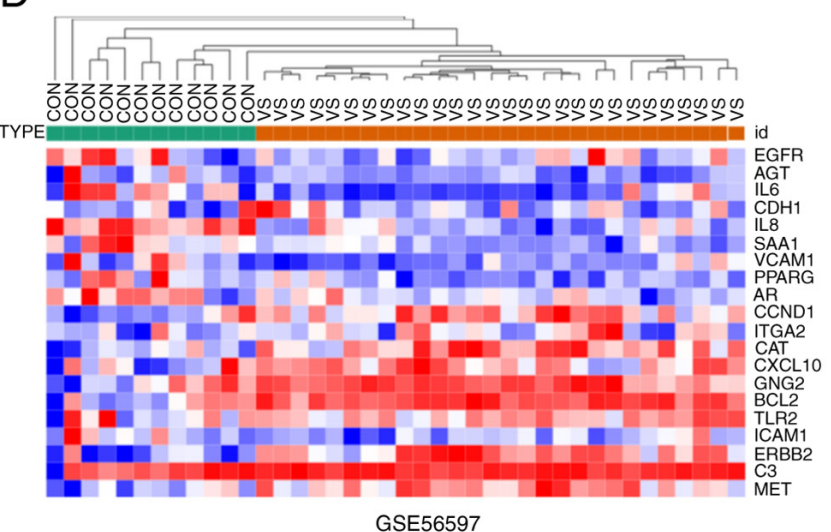

Figure 1. (A) Venn plot analysis results of differentially expressed genes. (B) Hub gene expression heat map of GSE39645. (C) Hub gene expression heat map of GSE54934. (D) Hub gene expression heat map of GSE56597.

AATTG; IL6 anti-sense, CCATCTTTGGAAGGTTCAGGT TG; and ITGA2 sense, CCTACAATGTTGGTCTCCCAGA; and ITGA2 anti-sense, AGTAACCAGTTGCCTTTTGGATT and GAPDH sense, GGAGCGAGATCCCTCCAAAAT.

Western blot analysis. Western blot analysis was performed to further verify the expression of BCL2, AGT, IL6 and ITGA2 in human Schwann cell line and schwannoma cell lines. Total protein extracts of tissues were obtained using RIPA buffer (Pierce; Thermo Fisher Scientific, Inc.). Protein concentration was determined using the Bradford method (Bio-Rad Laboratories, Inc.). Protein samples were separated using 10\% SDS-PAGE and transferred to PVDF membranes (Roche Applied Science). The membranes were preincubated with $5 \%$ skim milk in Tris-buffered saline (TBS) for $2 \mathrm{~h}$ at room temperature.

All cell lines were cultured in the same growth media. The protein samples from cultures were separated through electrophoresis. The target proteins in membranes were detected using primary antibodies to BCL2, AGT, IL6 and ITGA2, and incubated with the selected secondary antibodies. The following primary antibodies (EASYBIO) were diluted 1:1,000 in 1X TBS with 0.1\% Tween-20 (TBST), and the samples were incubated overnight at $4^{\circ} \mathrm{C}$ : Plant GST rabbit polyclonal (cat. no. be3158-50), phosphotyrosine mouse monoclonal (cat. no. be 3431) transferrin rabbit polyclonal (cat. no. be3357-100), ubiquitin mouse monoclonal (cat. no. be3601) and SPB (cat. no. wrab-48604) antibodies. The membranes were washed four times with TBST, horseradish peroxidase (HRP)-conjugated secondary antibodies, goat anti-rabbit IgG-HRP; cat. no. sc-2004; Santa Cruz Biotechnology, Inc.) were added, and the membranes were incubated for $1 \mathrm{~h}$ at room temperature. The samples were diluted 1:1,000 in TBST, and the hybridized bands were detected with an ECL detection kit (Thermo Fisher Scientific, Inc.) and Chemidoc XR image analyzer (Bio-Rad Laboratories, Inc.).

Following that, the membranes were visualized with an enhanced chemiluminescence detection system (Pierce; Thermo Fisher Scientific, Inc.). Western blot band intensities were analyzed with the National Institutes of Health Image 1.63 program (developed at the US National Institutes of Health; available at http://rsb.info.nih.gov/nih-image/).

Statistics. All statistic data were entered into SPSS 18.0 (SPSS Inc.) for analysis. ANOVA was used to analyze multiple comparison of RT-qPCR and Western blot analysis. Dunnett's t-test was performed as post hoc analysis. $\mathrm{P}<0.05$ was considered to indicate a statistically significant difference.

\section{Results}

Identification of DEGs. The series of data were analyzed separately by GeneSpring software, and DEGs were finally identified. A total of 4,025, 11,291 and 1,513 DEGs were identified from GSE54934, GSE56597 and GSE39645 
Table I. Functional and pathway enrichment analysis of upregulated and downregulated genes among three datasets.

\begin{tabular}{|c|c|c|c|c|c|}
\hline & Category & Term & Count & $\%$ & P-value \\
\hline \multirow[t]{20}{*}{ Upregulated } & GOTERM_BP_FAT & GO:0006955-immune response & 155 & 15.78 & $2.77 \times 10^{-15}$ \\
\hline & GOTERM_BP_FAT & GO:0031175-neuron projection development & 100 & 10.18 & $3.35 \times 10^{-15}$ \\
\hline & GOTERM_BP_FAT & GO:0061564-axon development & 66 & 6.72 & $5.55 \times 10^{-14}$ \\
\hline & GOTERM_BP_FAT & GO:0048666-neuron development & 108 & 11.00 & $9.34 \times 10^{-14}$ \\
\hline & GOTERM_BP_FAT & GO:0007155-cell adhesion & 160 & 16.29 & $1.54 \times 10^{-13}$ \\
\hline & GOTERM_CC_FAT & GO:0000323-lytic vacuole & 77 & 7.84 & $2.99 \times 10^{-13}$ \\
\hline & GOTERM_CC_FAT & GO:0005764-lysosome & 77 & 7.84 & $2.99 \times 10^{-13}$ \\
\hline & GOTERM_CC_FAT & GO:0005773-vacuole & 130 & 13.24 & $1.74 \times 10^{-12}$ \\
\hline & GOTERM_CC_FAT & GO:0044437-vacuolar part & 82 & 8.35 & $2.71 \times 10^{-9}$ \\
\hline & GOTERM_CC_FAT & GO:0005765-lysosomal membrane & 42 & 4.28 & $7.37 \times 10^{-8}$ \\
\hline & GOTERM_MF_FAT & GO:0008092-cytoskeletal protein binding & 77 & 7.84 & $2.27 \times 10^{-6}$ \\
\hline & GOTERM_MF_FAT & GO:0005102-receptor binding & 114 & 11.61 & $1.23 \times 10^{-5}$ \\
\hline & GOTERM_MF_FAT & GO:0098772-molecular function regulator & 109 & 11.10 & $1.94 \times 10^{-5}$ \\
\hline & GOTERM_MF_FAT & GO:0045499-chemorepellent activity & 9 & 0.92 & $4.91 \times 10^{-5}$ \\
\hline & GOTERM_MF_FAT & GO:0032395-MHC class II receptor activity & 7 & 0.71 & $6.67 \times 10^{-5}$ \\
\hline & KEGG_PATHWAY & hsa05150-Staphylococcus aureus infection & 22 & 2.24 & $2.69 \times 10^{-12}$ \\
\hline & KEGG_PATHWAY & hsa04514-cell adhesion molecules (CAMs) & 32 & 3.26 & $5.4 \times 10^{-10}$ \\
\hline & KEGG_PATHWAY & hsa04142-lysosome & 25 & 2.55 & $3.45 \times 10^{-7}$ \\
\hline & KEGG_PATHWAY & hsa04145-phagosome & 28 & 2.85 & $7.55 \times 10^{-7}$ \\
\hline & KEGG_PATHWAY & hsa05140-leishmaniasis & 16 & 1.63 & $2.57 \times 10^{-5}$ \\
\hline \multirow[t]{20}{*}{ Downregulated } & GOTERM_BP_FAT & GO:0040011-locomotion & 81 & 20.10 & $4.96 \times 10^{-13}$ \\
\hline & GOTERM_BP_FAT & GO:0016477-cell migration & 69 & 17.12 & $5.67 \times 10^{-13}$ \\
\hline & GOTERM_BP_FAT & GO:0048870-cell motility & 74 & 18.36 & $6.35 \times 10^{-13}$ \\
\hline & GOTERM_BP_FAT & GO:0051674-localization of cell & 74 & 18.36 & $6.35 \times 10^{-13}$ \\
\hline & GOTERM_BP_FAT & GO:0072359-circulatory system development & 60 & 14.89 & $8.12 \times 10^{-13}$ \\
\hline & GOTERM_CC_FAT & GO:0005615-extracellular space & 83 & 20.60 & $5.27 \times 10^{-15}$ \\
\hline & GOTERM_CC_FAT & GO:0031012-extracellular matrix & 44 & 10.92 & $5.55 \times 10^{-13}$ \\
\hline & GOTERM_CC_FAT & GO:0005576-extracellular region & 169 & 41.94 & $1.45 \times 10^{-12}$ \\
\hline & GOTERM_CC_FAT & GO:0005578-proteinaceous extracellular matrix & 34 & 8.44 & $1.42 \times 10^{-11}$ \\
\hline & GOTERM_CC_FAT & GO:0044421-extracellular region part & 143 & 35.48 & $2.47 \times 10^{-10}$ \\
\hline & GOTERM_MF_FAT & GO:0005102-receptor binding & 67 & 16.63 & $1.15 \times 10^{-8}$ \\
\hline & GOTERM_MF_FAT & GO:0005539 glycosaminoglycan binding & 21 & 5.21 & $3.56 \times 10^{-8}$ \\
\hline & GOTERM_MF_FAT & GO:0005509-calcium ion binding & 38 & 9.43 & $1.35 \times 10^{-6}$ \\
\hline & GOTERM_MF_FAT & GO:0008201-heparin binding & 16 & 3.97 & $2.57 \times 10^{-6}$ \\
\hline & GOTERM_MF_FAT & GO:0008237-metallopeptidase activity & 17 & 4.22 & $4.86 \times 10^{-6}$ \\
\hline & KEGG_PATHWAY & hsa05144-malaria & 10 & 2.48 & $3.45 \times 10^{-6}$ \\
\hline & KEGG_PATHWAY & hsa04024-cAMP signaling pathway & 16 & 3.97 & $1.65 \times 10^{-4}$ \\
\hline & KEGG_PATHWAY & hsa04060-cytokine-cytokine receptor interaction & 17 & 4.22 & $2.68 \times 10^{-4}$ \\
\hline & KEGG_PATHWAY & hsa05200-pathways in cancer & 23 & 5.71 & $4.45 \times 10^{-4}$ \\
\hline & KEGG_PATHWAY & hsa04020-calcium signaling pathway & 14 & 3.47 & $6.81 \times 10^{-4}$ \\
\hline
\end{tabular}

GO, Gene Ontology; KEGG, Kyoto Encyclopedia of Genes and Genomes.

datasets. In GSE54934, there were 1,225 upregulated and 2,800 downregulated DEGs; in GSE56597, the numbers of upand downregulated DEGs were 7,687 and 3,604, respectively; in GSE39645, 1,101 genes were upregulated and 412 were downregulated. The results of Venn plot screened 1386 mutual DEGs, as presented in Fig. 1A and Table S1, and heatmap of DEGs are shown in Fig. 1B-D.
Functional and pathway enrichment analysis. To gain further insight into the molecular mechanism of VA, the mutual DEGs were further explored for functional and pathway enrichment analysis, which are shown in Table I and Fig. 2A and B. In biological processes (BP), the upregulated genes were mainly enriched in immune response, cell adhesion and neuron development, while downregulated genes were involved in 
A

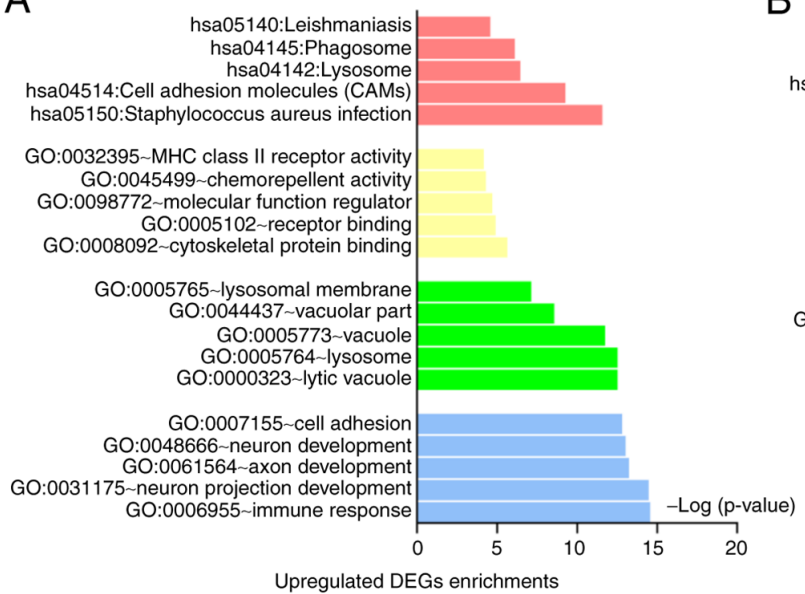

B

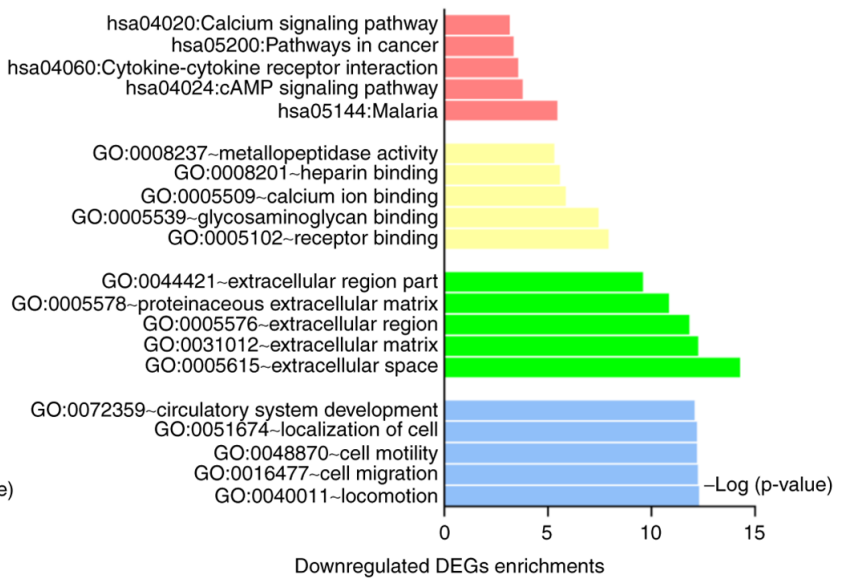

Figure 2. (A) Functional and pathway enrichment analysis of upregulated genes. (B) Functional and pathway enrichment analysis of downregulated genes. DEGS, differentially expressed genes.
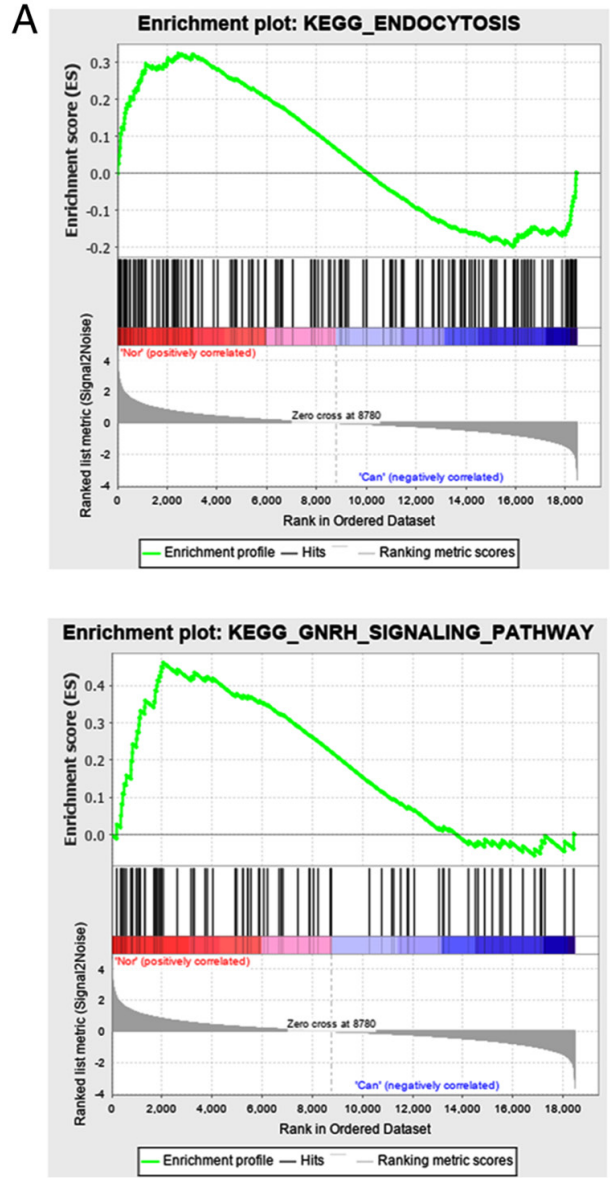

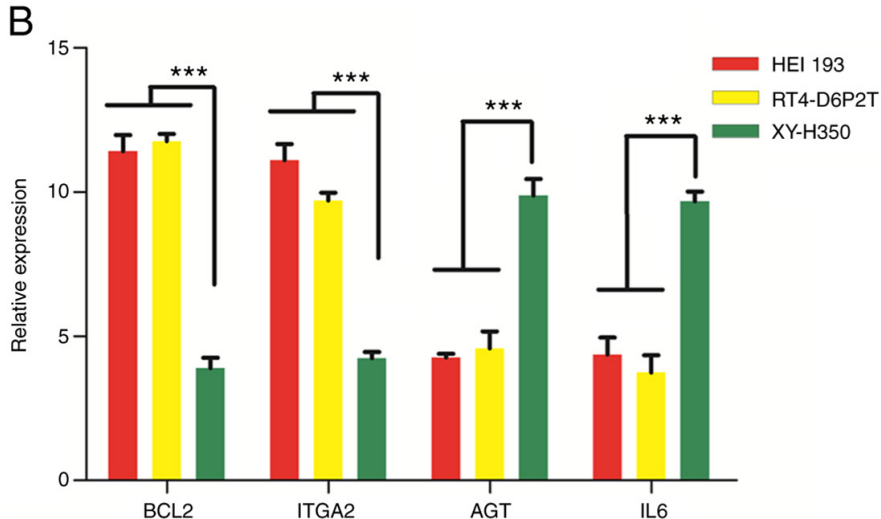

C

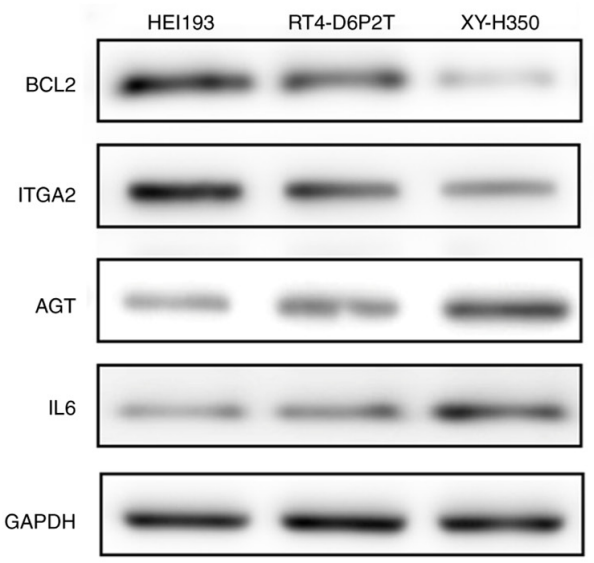

Figure 3. (A) Gene Set Enrichment Analysis of mutual differentially expressed genes among three datasets. (B) Results of reverse transcription-quantitative PCR analysis. ${ }^{* * *} \mathrm{P}<0.001$. (C) Results of western blot analysis.

locomotion, extracellular space, cell motility, localization of cell and circulatory system development. For cell component (CC), the upregulated genes were mainly associated with lysosome, lysosomal membrane and vacuolar part, whereas the downregulated genes were associated with extracellular space, extracellular matrix and extracellular region. In molecular function (MF), the upregulated genes were mainly enriched in cytoskeletal protein binding, receptor binding and chemorepellent activity, while the downregulated in receptor binding, calcium ion binding and heparin binding. Moreover, as shown in Fig. 3A, several KEGG pathways were overrepresented, which are cell adhesion molecules (CAMs), phagosome, lysosome, simultaneously, malaria and cAMP signaling pathway. 
Table II. Detailed information of the hub genes among three datasets.

\begin{tabular}{lcc}
\hline Gene symbol & Degree & Betweenness Centrality \\
\hline EGFR & 120 & 0.10487979 \\
ITGA2 & 104 & 0.08396193 \\
AGT & 89 & 0.040633 \\
IL6 & 84 & 0.04045457 \\
CDH1 & 80 & 0.04385459 \\
BCL2 & 80 & 0.04821594 \\
IL8 & 79 & 0.0255738 \\
ICAM1 & 78 & 0.02813842 \\
ERBB2 & 71 & 0.02459344 \\
CCND1 & 65 & 0.03077167 \\
MET & 60 & 0.03282732 \\
CAT & 55 & 0.04106918 \\
CXCL10 & 54 & 0.00806233 \\
GNG2 & 53 & 0.00652289 \\
SAA1 & 53 & 0.00540032 \\
VCAM1 & 52 & 0.01110284 \\
PPARG & 51 & 0.02454711 \\
AR & 50 & 0.02629149 \\
C3 & 50 & 0.01353721 \\
TLR2 & 50 & 0.01524084 \\
\hline
\end{tabular}

Module screening from the PPI network. The previous 1386 mutual DEGs among the three datasets were analyzed using PPI network, and the hub genes were screened with degrees $\geq 50$ based on the STRING database. Altogether, 20 genes were identified as hub genes, which were listed in Table II, including EGFR, ITGA2, AGT, IL6, CDH1, BCL2, IL8, ICAM1, ERBB2, CCND1, MET, CAT, CXCL10, GNG2, SAA1, VCAM1, PPARG, ARC3 and TLR2. Among the aforementioned genes, EGFR showed the highest degree, which was 120 . The top three significant modules were selected and shown in Fig. 4. Functional annotation of the module genes is shown in Table III. The genes of module 1 were associated with cellular structure, including G-protein coupled with receptor signaling pathway, inflammatory response and chemokine-mediated signaling pathway. Genes of module 2 were associated with type I interferon signaling pathway, interferon- $\gamma$-mediated signaling pathway and defense response to virus. Genes of module 3 were associated with type I interferon signaling pathway, interferon- $\gamma$-mediated signaling pathway and defense response to virus.

Validation of common hub genes by RT-qPCR. To validate the expression of BCL2, AGT, IL6, and ITGA2 in human schwannoma cells, RT-qPCR was performed. Fig. 3B showed significant differences in the expression levels of these genes among human Schwann cells, RT4-D6P2T cell line and HEI193 cell line. BCL2 and ITGA2 genes were consistently expressed higher in RT4-D6P2T cells and HEI193 cells compared with normal Schwann cells $(\mathrm{P}<0.05)$. AGT and IL6, on the contrary, showed lower expression in schwannoma cell lines with human Schwann cells. Moreover, among the schwannoma cell lines, the levels of expression of the BCL2, AGT, IL6, and ITGA2 genes were slightly different.

Further confirmation of common hub genes by western blotting. Western blot analysis was conducted to verify the expression of BCL2, AGT, IL6 and ITGA2 in human Schwann cells, RT4-D6P2T cell line and HEI193 cell line. As presented in Fig. 3C, the protein levels of BCL2 and ITGA2 was distinctly expressed higher in RT4-D6P2T cells and HEI193 cells compared with normal Schwann cells, while AGT and IL6 had lower expression in schwannoma cell lines compared with human Schwann cells. The result of western blotting analysis was consistent with the RT-qPCR result.

\section{Discussion}

VS is a product of the cumulative genetic, epigenetic, somatic and endocrine aberrations. Despite advances in treatment methods, including surgical resection, stereotactic radiotherapy and long-term follow-up observation, the prognosis of VS remained poor over the past decades, mainly due to the lack of effective diagnosis and therapy (13). Therefore, to comprehensively perceive the molecular mechanisms, etiological factors and pathogenesis of VS is vital for the improvement of diagnosis, therapy and prognosis. Recently, microarray technology has been developed rapidly and widely used to reveal the general genetic alteration in the progression of disease, which enables the identification of targets for diagnosis, therapeutic and prognosis of tumors.

In the present study, 49 VS samples and 31 formal samples were extracted from mRNA microarray dataset GSE39645, GSE454934 and GSE56597 for gene expression data. A total of 1,088 DEGs, 1,744 DEGs and 983 DEGs were identified respectively. A total of 1,386 mutual DEGs among the three datasets were identified by Venn plot. Cumulative evidences proved that these DEGs were expressed abnormally and, significantly, and played an important role in the pathogenesis of VS, which could be used as diagnosis, treatment and prognosis markers in the future. In order to better perceive the interactions of DEGs, GO and KEGG pathway analysis were further performed.

Based on GO analysis of abnormal expression genes, the upregulated genes were mainly associated with hyperplasia, including axon development, cell adhesion, cytoskeletal protein binding, molecular function regulator and MHC class II receptor activity, which were associated with cancer. The downregulated genes were primarily enriched in biological information transfer, such as cell migration, cell motility, circulatory system development, calcium ion binding and extracellular region part. The communication between cells and its surrounding environment is bidirectional.

Microenvironments have been shown to be important for normal tissue homeostasis and tumor tissue growth. The interaction of Schwann cells and axons with the surrounding cells is one of the key factors in schwannoma, which is consistent with the result of the GO analysis. In addition, schwannoma is closely associated with the abnormity of macrophage, indicating that anti-inflammatory therapy may be with potential curative effect of schwannoma (14). 


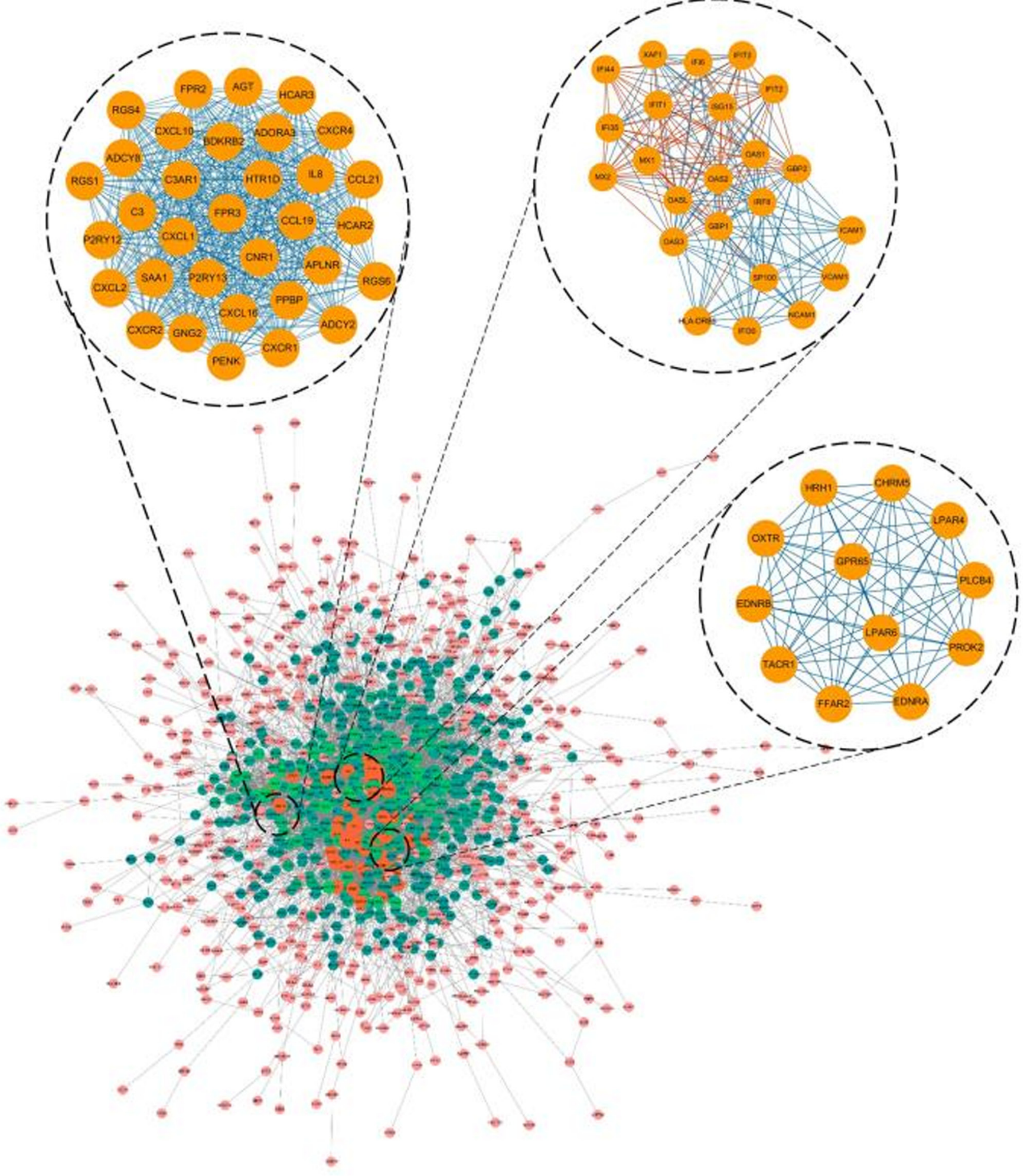

Figure 4. Top three modules from the protein-protein interaction network.

Furthermore, the results of KEGG analysis revealed that the mutual up- and downregulated DEGs were mainly enriched in cell adhesion molecules (CAMs), phagosome and cAMP signaling pathway. CAMs not only play an important role in normal tissue growth and development, participating in proliferation and differentiation, signal transduction of cells, but also in the biological behavior of tumor tissues, participating in the occurrence, invasion and metastasis of tumor. Neural cell adhesion molecule (NCAM) is a marker of Schwann cells. Some studies have shown that NCAM was overexpressed in Schwann cells of patients with VS. The degree of CAM expression can help us distinguish between benign and malignant schwannomas (15). E-cadherin is a calcium-dependent CAM, which is an essential protein for 
Table III. Functional and pathway enrichment analysis of the module's genes.

\begin{tabular}{|c|c|c|c|c|c|}
\hline Modules & Term & Count & $\%$ & P-value & Genes \\
\hline \multirow[t]{3}{*}{1} & $\begin{array}{l}\text { G-protein coupled receptor } \\
\text { signaling pathway (BP) }\end{array}$ & 20 & 62.50 & $4.31 \times 10^{-17}$ & $\begin{array}{l}\text { CXCL1, C3AR1, C3, CXCL2, CXCR1, CCL19, FPR2, } \\
\text { BDKRB2, CXCL10, P2RY12, APLNR, RGS1, PPBP, } \\
\text { CXCR4, CCL21, AGT, RGS6, GNG2, HCAR3, HCAR2 }\end{array}$ \\
\hline & Inflammatory response (BP) & 14 & 43.75 & $4.60 \times 10^{-14}$ & $\begin{array}{l}\text { CXCL1, C3AR1, C3, CXCL2, CXCR1, CCL19, CXCR2, } \\
\text { FPR3, BDKRB2, FPR2, CXCL10, PPBP, CCL21, CXCR4 }\end{array}$ \\
\hline & $\begin{array}{l}\text { Chemokine-mediated } \\
\text { signaling pathway (BP) }\end{array}$ & 9 & 28.12 & $4.97 \times 10^{-13}$ & $\begin{array}{l}\text { CXCL1, PPBP, CXCR4, CCL21, CXCL2, CXCR1, CCL19, } \\
\text { CXCR2, CXCL10 }\end{array}$ \\
\hline \multirow[t]{3}{*}{2} & $\begin{array}{l}\text { Type I interferon signaling } \\
\text { pathway (BP) }\end{array}$ & 16 & 69.56 & $1.47 \times 10^{-32}$ & $\begin{array}{l}\text { SP100, OAS3, OAS1, OAS2, IFI35, IFIT3, IFIT2, OASL, } \\
\text { IFIT1, ISG15, IRF8, XAF1, MX1, MX2, GBP2, IFI6 }\end{array}$ \\
\hline & $\begin{array}{l}\text { Interferon- } \gamma \text {-mediated } \\
\text { signaling pathway (BP) }\end{array}$ & 13 & 56.52 & $7.65 \times 10^{-24}$ & $\begin{array}{l}\text { NCAM1, VCAM1, ICAM1, OASL, SP100, IRF8, OAS3, } \\
\text { HLA-DRB5, IFI30, OAS1, OAS2, GBP2, GBP1 }\end{array}$ \\
\hline & $\begin{array}{l}\text { Defense response to virus } \\
\text { (BP) }\end{array}$ & 11 & 47.82 & $3.69 \times 10^{-15}$ & $\begin{array}{l}\text { IFIT3, IFIT2, IFIT1, OASL, ISG15, OAS3, OAS1, OAS2, } \\
\text { MX1, MX2, GBP1 }\end{array}$ \\
\hline \multirow[t]{3}{*}{3} & $\begin{array}{l}\text { Type I interferon signaling } \\
\text { pathway (BP) }\end{array}$ & 16 & 69.56 & $1.47 \times 10^{-32}$ & $\begin{array}{l}\text { SP100, OAS3, OAS1, OAS2, IFI35, IFIT3, IFIT2, OASL, } \\
\text { IFIT1, ISG15, IRF8, XAF1, MX1, MX2, GBP2, IFI6 }\end{array}$ \\
\hline & $\begin{array}{l}\text { Interferon- } \gamma \text {-mediated } \\
\text { signaling pathway (BP) }\end{array}$ & 13 & 56.52 & $7.65 \times 10^{-24}$ & $\begin{array}{l}\text { NCAM1, VCAM1, ICAM1, OASL, SP100, IRF8, OAS3, } \\
\text { HLA-DRB5, IFI30, OAS1, OAS2, GBP2, GBP1 }\end{array}$ \\
\hline & $\begin{array}{l}\text { Defense response to virus } \\
\text { (BP) }\end{array}$ & 11 & 47.82 & $3.69 \times 10^{-15}$ & $\begin{array}{l}\text { IFIT3, IFIT2, IFIT1, OASL, ISG15, OAS3, OAS1, OAS2, } \\
\text { MX1, MX2, GBP1 }\end{array}$ \\
\hline
\end{tabular}

$\mathrm{BP}$, biological process.

metastasis of multiple models of breast cancer and a lack of E-cadherin can induce apoptosis of cells (16). E-cadherin is expressed in some schwannomas, especially in VS (14). Therefore, it was speculated that E-cadherin is closely associated with the metastasis of tumor cells in VS, and molecular strategies to inhibit E-cadherin-mediated tumor cell metastasis may have potential effect in the treatment of VS. Phagosome promotes immunosuppression through induction of macrophages with increasing expression of PD-L1 (17). A study has shown that PD-L1 is highly expressed in cells of VS, and adaptive resistance to cellular immunity plays an important role in tumor immune microenvironment of schwannoma (18). Therefore, it is postulated that immunotherapy is expected to be a new treatment method for VS.

Aims to screen hub genes among DEGs were identified in previous studies, therefore, mutual DEGs were analyzed with PPI network based on the STRING database, and 20 genes were selected with high degrees, such as BCL2, AGT, IL6 and ITGA2.

BCL2, an apoptosis regulator, located in chromosome $1 q 42.2$, the epigenetic inactivation of which was caused by promoter hypermethylation may lead to mutations in K-ras and p53, a critical process that initiates tumorigenesis (19). A study by $\mathrm{Ni}$ et al (20) proved that co-overexpression of VEGF and Bcl-2 inhibits the oxygen and glucose deprivation, inducing the apoptosis of mesenchymal stem cells. Edison et al (21) reported that the mutation of BCL2 increased stability and was more potent in the protection against apoptosis. It has also been reported that BCL2 is associated with certain malignancies including gastrin, hepatocellular carcinoma, salivary adenoid cystic carcinoma, and diffuse large B cell lymphoma (22). In addition, it was demonstrated that BCL2 is associated with the migration and invasion of human osteosarcoma and breast cancer, therefore, this could be a significant marker for tumor aggressiveness (12,23-26). Furthermore, it has been reported that bufalin synergized with AKT inhibitor LY294002 to induce the apoptosis of lung cancer A549 cells, which was associated with the upregulation of the downregulation of BCL2, which indicates that BCL2 is a potential therapeutic target for VS, and bufalin may be a potential treatment regimen (27).

AGT, encoding angiotensinogen and angiotensin-(1-7) plays a role in metabolic pathways associated with cell death and cell survival in human endothelial cells. As a vital progenitor, the augmented AGT accelerated vascular remodeling. It is universally known that the circulation via blood vessels is a significant process in tumor metastasis (28-30). AGT is markedly associated with the initiation and progression of brain, lung, ovary and renal cell cancer $(31,32)$. In addition, the serum AGT is also associated with the prognosis of metastatic colorectal cancer (33).

IL6, located on chromosome 7p15.3, as a pleiotropic cytokine, plays an important role in immune regulation, hematopoiesis, inflammation and oncogenesis, and controls the survival, growth, differentiation and effector function of tissues and cells, and functions in inflammation and maturation of B cells. It was reported that Il-6 is associated with various malignancies including melanoma, breast cancer, gastric cancer, colorectal cancer and prostate cancer (34). IL6 enhances tumorigenesis, promotes melanoma progression and strengthens tumorigenic capabilities (35-39). Furthermore, it 
is a diagnostic biomarker for gastric cancer and a prognostic biomarker in metastatic colorectal cancer (40). In addition, the biological mechanisms of IL-6 in acoustic neuroma were reported to be associated with the stimulation of cellular proliferation, and may be the potential therapeutic targets in VS $(41,42)$.

ITGA2 is located on chromosome 5q11.2. As a part of cell-matrix adhesion, ITGA2 interacts selectively and non-covalently with amyloid- $\beta$ peptide/protein and/or its precursor. ITGA2 is the attachment for a cell, either to another cell or to an underlying substrate such as the extracellular matrix, via an integrin, a heterodimeric adhesion receptor formed by the non-covalent association of particular $\alpha$ and $\beta$ subunits. It is known that the movement of normal cells is also controlled by contact inhibition, which largely depends on adhesion molecules such as integrins that prevents aberrant cell migration (43). ITGA2 has been pointed that it is associated with the occurrence, development and metastasis of breast cancer, nasopharyngeal carcinoma, primary colon tumors, cervical cancer and liver cancer. It also has been reported that it might be a therapeutic target for gastric cancer and osteosarcoma. Besides, ITGA2 may be a prognosis biomarker for breast cancer patients. All types of evidence suggest that in the early process of cancer metastasis, ITGA2 reduction helps cancer cells to detach the primary cancer and in late phase cancer, ITGA2 recovery helps cancer cells to locate in lymph node and distant organs (44-48).

Aiming to verify the results of bioinformatics analysis, RT-qPCR and western blot analysis of the aforementioned hub genes were performed. The results indicated that expression levels of BCL2 and ITGA2 in schwannoma samples were significantly higher compared with normal Schwann cells $(\mathrm{P}<0.05)$, while AGT and IL6 were expressed lower in schwannoma cell lines compared with normal Schwann cells, which is consistent with DEG identification and heatmaps. In addition BCL2, AGT, IL6 and ITGA2 were revealed in VS for the first time, which provides verification of the associations among these genes and VS for further investigations. The other hub genes also mean precise diagnosis biomarkers, potential treatment targets, and prognosis markers for patients with VS, as well as DEGs.

The functional annotation and enrichment of module genes were also performed, and enriched function analysis and it revealed that genes in module 1 were primarily associated with inflammatory response, chemokine-mediated signaling pathway and G-protein coupled with receptor signaling pathway. Inflammatory response is closely associated with tumor, studies have proved that inflammatory environment affects gastric cancer initiation and metastasis via epithelial-mesenchymal transition (49). Tumor-associated inflammation can promote tumorigenesis and metastasis through angiogenesis and metastasis, which also turn reduced the sensitivity of tumor to chemotherapy drugs. The genes of modules 2 and 3 were associated with type I interferon signaling pathway, interferon- $\gamma$-mediated signaling pathway and defense response to virus. Type I interferon is significant for defense against viruses via the induction of antiviral effector molecules that are encoded by IFN-stimulated genes (50). Due to the immunosuppressive state of patients with schwannoma (51), T-lymphocytes-secreted interferon is decreased, which is conducive to the growth and reproduction of tumor cells and reduces the resistance to virus (52). Insufficient secretion of interferon is highly susceptible to virus infection (53).

Conclusion. A total of 4,025, 11,291 and 1,513 DEGs were identified from GSE54934, GSE56597 and GSE39645 datasets, respectively, and there are 1,386 mutual DEGs among these three datasets. The GO and KEGG analysis showed that enriched functions and pathways in upregulated genes are mainly associated with cell division, mitotic nuclear division, transition of mitotic cell cycle, microtubule, and microtubule motor activity, while downregulated genes tend to enrich in biological information transfer, including chemical synaptic transmission, neurotransmitter transport, synaptic vesicle membrane, GABA-A receptor activity and calcium ion binding. BCL2, AGT, IL6 and ITGA2 were screened as main hub genes, among which BCL2 and ITGA2 were significantly higher expressed in VS cells compared with human normal glial cells, while the expression of AGT and IL6 was lower in VS cells compared with human normal glial cells. In addition, the present study provided the mechanisms and molecules that were involved in the interaction between tumor microenvironment and inflammatory response, which may be targets for anticancer interventions.

\section{Acknowledgements}

Not applicable.

\section{Funding}

This study was supported by grants from the National Natural ScienceFoundation of China (grantnos.81672505 and 81772684), the S\&T Development Planning Program of Jilin Province (grant nos. 20160101086JC, 20160312017ZG and 20180101152JC), the Jilin Provincial Education Department '13th Five-Year' Science and Technology Project (grant no. JJKH20180191KJ) and the Interdisciplinary Innovation Project of The First Hospital of Jilin University (grant no. JDYYJC001).

\section{Availability of data and materials}

The datasets generated and/or analyzed during the current study are available in the Gene Ontology (GO), Kyoto Encyclopedia of Genes and Genomes (KEGG), protein-protein interaction (PPI) repository, Gene Expression Omnibus (GEO, http://www.ncbi.nlm.nih.gov/geo) database of National Center of Biotechnology Information (NCBI) (http://www. ncbi.nlm.nih.gov/geo/) and The Database for Annotation, Visualization and Integrated Discovery (DAVID; http://david. abcc.ncifcrf.gov/). Specific datasets were obtainted from GEO (GSE54934, https://www.ncbi.nlm.nih.gov/geo/query/acc. cgi?acc=GSE54934; GSE39645, https://www.ncbi.nlm.nih. gov/geo/query/acc.cgi?acc=GSE39645; GSE56597, https:// www.ncbi.nlm.nih.gov/geo/query/acc.cgi?acc=GSE56597).

\section{Authors' contributions}

BW and GD designed the experiments, planned the experiment flow and revised the manuscript. YZ and JW wrote 
the manuscript, consulted literature, screened the raw data and performed the Venn diagram analysis. JR and XW performed GO and KEGG analysis. SJ and SZ conducted heat map analysis and PPI network analysis. ZZ, CS and $\mathrm{JL}$ analyzed the data and collated the data into figures and tables. GZ analyzed sequencing data. LZ revised the manuscript, tables and figures, and performed western blot analysis and revised it critically for important intellectual content. All authors read and approved the final manuscript. YZ and JW confirm the authenticity of all the raw data.

\section{Ethics approval and consent to participate}

Not applicable.

\section{Patient consent for publication}

Not applicable.

\section{Competing interests}

The authors declare that they have no competing interests.

\section{References}

1. Ramaswamy AT and Golub JS: Management of vestibular schwannomas for the radiologist. Neuroimaging Clin N Am 29: 173-182, 2019.

2. Mahaley MS Jr, Mettlin C, Natarajan N, Laws ER Jr and Peace BB: Analysis of patterns of care of brain tumor patients in the United States: A study of the brain tumor section of the AANS and the CNS and the commission on cancer of the ACS Clin Neurosurg 36: 347-352, 1990.

3. Basu S, Youngs R and Mitchell-Innes A: Screening for vestibular schwannoma in the context of an ageing population. J Laryngol Otol 133: 640-649, 2019.

4. Kaul V and Cosetti MK: Management of vestibular schwannoma (Including NF2): Facial nerve considerations. Otolaryng Clin North Am 51: 1193-1212, 2018.

5. Martini A, Marioni G, Zanoletti E, Cappellesso R, Stramare R, Fasanaro E, Faccioli C, Giacomelli L, Denaro L, D'Avella D, et al: YAP, TAZ and AREG expression in eighth cranial nerve schwannoma. Int J Biol Markers 32: e319-e324, 2017.

6. Zhang Z, Wang Z, Sun L, Li X, Huang Q, Yang T and Wu H: Mutation spectrum and differential gene expression in cystic and solid vestibular schwannoma. Genet Med 16: 264-270, 2014.

7. Torres-Martin M, Lassaletta L, Isla A, De Campos JM, Pinto GR, Burbano RR, Castresana JS, Melendez B and Rey JA: Global expression profile in low grade meningiomas and schwannomas shows upregulation of PDGFD, CDH1 and SLIT2 compared to their healthy tissue. Oncol Rep 32: 2327-2334, 2014.

8. Torres-Martin M, Lassaletta L, San-Roman-Montero J, De Campos JM, Isla A, Gavilan J, Melendez B, Pinto GR, Burbano RR, Castresana JS and Rey JA: Microarray analysis of gene expression in vestibular schwannomas reveals SPP1/MET signaling pathway and androgen receptor deregulation. Int J Oncol 42: 848-862, 2013.

9. Torres-Martín M, Lassaletta L, de Campos JM, Isla A Pinto GR, Burbano RR, Melendez B, Castresana JS and Rey JA: Genome-wide methylation analysis in vestibular schwannomas shows putative mechanisms of gene expression modulation and global hypomethylation at the HOX gene cluster. Genes Chromosomes Cancer 54: 197-209, 2015.

10. Shannon P, Markiel A, Ozier O, Baliga NS, Wang JT, Ramage D, Amin N, Schwikowski B and Ideker T: Cytoscape: A software environment for integrated models of biomolecular interaction networks. Genome Res 13: 2498-2504, 2003.

11. Otasek D, Morris JH, Bouças J, Pico AR and Demchak B Cytoscape automation: Empowering workflow-based network analysis. Genome Biol 20: 185, 2019.
12. Leng J, Song Q, Zhao Y and Wang Z: miR-15a represses cancer cell migration and invasion under conditions of hypoxia by targeting and downregulating $\mathrm{Bcl} 2$ expression in human osteosarcoma cells. Int J Oncol 52: 1095-1104, 2018.

13. Mehrian-Shai R, Freedman S, Shams S, Doherty J, Slattery W, Hsu NY, Reichardt JK, Andalibi A and Toren A: Schwannomas exhibit distinct size-dependent gene-expression patterns. Future Oncol 11: 1751-1758, 2015.

14. Schulz A, Büttner R, Hagel C, Baader SL, Kluwe L, Salamon J, Mautner VF, Mindos T, Parkinson DB, Gehlhausen JR, et al: The importance of nerve microenvironment for schwannoma development. Acta Neuropathol 132: 289-307, 2016.

15. Roche PH, Figarella-Branger D, Daniel L, Bianco N, Pellet W and Pellissier JF: Expression of cell adhesion molecules in normal nerves, chronic axonal neuropathies and Schwann cell tumors. J Neurol Sci 151: 127-133, 1997.

16. Padmanaban V, Krol I, Suhail Y, Szczerba BM, Aceto N, Bader JS and Ewald AJ: E-cadherin is required for metastasis in multiple models of breast cancer. Nature 573: 439-444, 2019.

17. Wen ZF, Liu H, Gao R, Zhou M, Ma J, Zhang Y, Zhao J, Chen Y, Zhang T, Huang F, et al: Tumor cell-released autophagosomes (TRAPs) promote immunosuppression through induction of M2-like macrophages with increased expression of PD-L1. J Immunother Cancer 6: 151, 2018.

18. Wang S, Liechty B, Patel S, Weber JS, Hollmann TJ, Snuderl M and Karajannis MA: Programmed death ligand 1 expression and tumor infiltrating lymphocytes in neurofibromatosi type 1 and 2 associated tumors. J Neurooncol 138: 183-190, 2018.

19. Liang B, Li C and Zhao J: Identification of key pathways and genes in colorectal cancer using bioinformatics analysis. Med Oncol 33: 111, 2016.

20. Ni X, Ou C, Guo J, Liu B, Zhang J, Wu Z, Li H and Chen M Lentiviral vector-mediated co-overexpression of VEGF and Bcl-2 improves mesenchymal stem cell survival and enhances paracrine effects in vitro. Int J Mol Med 40: 418-426, 2017.

21. Edison N, Curtz Y, Paland N, Mamriev D, Chorubczyk N, Haviv-Reingewertz T, Kfir N, Morgenstern D, Kupervaser M, Kagan J, et al: Degradation of Bcl-2 by XIAP and ARTS promotes apoptosis. Cell Rep 21: 442-454, 2017.

22. Ozretic P, Alvir I, Sarcevic B, Vujaskovic Z, Rendic-Miocevic Z, Roguljic A and Beketic-Oreskovic L: Apoptosis regulator Bcl-2 is an independent prognostic marker for worse overall survival in triple-negative breast cancer patients. Int J Biol Markers 33: 109-115, 2018.

23. Yan F, Wang C, Li T, Cai W and Sun J: Role of miR-21 in the growth and metastasis of human salivary adenoid cystic carcinoma. Mol Med Rep 17: 4237-4244, 2018.

24. Butler MJ and Aguiar RCT: Biology informs treatment choices in diffuse large B cell lymphoma. Trends Cancer 3: 871-882, 2017.

25. Feng L, Zhu J, Sun W, Zhao J and Liu Y: Expressions of gastrin and apoptosis-associated proteins involved in mitochondrial pathway in gastric cancer tissues and the clinical significance. Xi Bao Yu Fen Zi Mian Yi Xue Za Zhi 33: 1557-1561, 2017 (In Chinese).

26. You ML, Chen YJ, Chong QY, Wu MM, Pandey V, Chen RM, Liu L, Ma L, Wu ZS, Zhu T and Lobie PE: Trefoil factor 3 mediation of oncogenicity and chemoresistance in hepatocellular carcinoma is AKT-BCL-2 dependent. Oncotarget 8: 39323-39344, 2017.

27. Liu F, Tong D, Li H, Liu M, Li J, Wang Z and Cheng X: Bufalin enhances antitumor effect of paclitaxel on cervical tumorigenesis via inhibiting the integrin $\alpha 2 / \beta 5 / \mathrm{FAK}$ signaling pathway. Oncotarget 7: 8896-8907, 2016.

28. Sato Y, Kubo T, Morimoto K, Yanagihara K and Seyama T: High mannose-binding Pseudomonas fluorescens lectin (PFL) downregulates cell surface integrin/EGFR and induces autophagy in gastric cancer cells. BMC Cancer 16: 63, 2016

29. Wu SJ, Soulez M, Yang YH, Chu CS, Shih SC, Hebert MJ, Kuo MC and Hsieh YJ: Local augmented angiotensinogen secreted from apoptotic vascular endothelial cells is a vital mediator of vascular remodelling. PLoS One 10: e0132583, 2015.

30. Meinert C, Gembardt F, Bohme I, Tetzner A, Wieland T, Greenberg B and Walther T: Identification of intracellular proteins and signaling pathways in human endothelial cells regulated by angiotensin-(1-7). J Proteomics 130: 129-139, 2016.

31. Citron M, Decker R, Chen S, Schneider S, Graver M, Kleynerman L, Kahn LB, White A, Schoenhaus M and Yarosh D: O6-methylguanine-DNA methyltransferase in human normal and tumor tissue from brain, lung, and ovary. Cancer Res 51: 4131-4134, 1991. 
32. Deckers IA, van den Brandt PA, van Engeland $M$, van Schooten FJ, Godschalk RW, Keszei AP and Schouten LJ: Polymorphisms in genes of the renin-angiotensin-aldosterone system and renal cell cancer risk: Interplay with hypertension and intakes of sodium, potassium and fluid. Int J Cancer 136 : 1104-1116, 2015.

33. Martin P, Noonan S, Mullen MP, Scaife C, Tosetto M, Nolan B, Wynne K, Hyland J, Sheahan K, Elia G, et al: Predicting response to vascular endothelial growth factor inhibitor and chemotherapy in metastatic colorectal cancer. BMC Cancer 14 887, 2014.

34. Deng G, Kakar S, Okudiara K, Choi E, Sleisenger MH and Kim YS: Unique methylation pattern of oncostatin $\mathrm{m}$ receptor gene in cancers of colorectum and other digestive organs. Clin Cancer Res 15: 1519-1526, 2009.

35. Sameni M, Cavallo-Medved D, Franco OE, Chalasani A, Ji K, Aggarwal N, Anbalagan A, Chen X, Mattingly RR, Hayward SW and Sloane BF: Pathomimetic avatars reveal divergent roles of microenvironment in invasive transition of ductal carcinoma in situ. Breast Cancer Res 19: 56, 2017.

36. Chen $\mathrm{C}$ and Zhang X: IRE1 $\alpha-\mathrm{XBP} 1$ pathway promotes melanoma progression by regulating IL-6/STAT3 signaling. J Transl Med 15: 42, 2017.

37. Ortiz-Montero P, Londono-Vallejo A and Vernot JP: Senescence-associated IL-6 and IL-8 cytokines induce a self- and cross-reinforced senescence/inflammatory milieu strengthening tumorigenic capabilities in the MCF-7 breast cancer cell line. Cell Commun Signal 15: 17, 2017.

38. Zhou J, Zhang C, Pan J, Chen L and Qi ST: Interleukin6 induces an epithelialmesenchymal transition phenotype in human adamantinomatous craniopharyngioma cells and promotes tumor cell migration. Mol Med Rep 15: 4123-4131, 2017.

39. Albino D, Civenni G, Rossi S, Mitra A, Catapano CV and Carbone GM: The ETS factor ESE3/EHF represses IL-6 preventing STAT3 activation and expansion of the prostate cancer stem-like compartment. Oncotarget 7: 76756-76768, 2016

40. Thomsen M, Kersten C, Sorbye H, Skovlund E, Glimelius B, Pfeiffer P, Johansen JS, Kure EH, Ikdahl T, Tveit KM, et al: Interleukin-6 and C-reactive protein as prognostic biomarkers in metastatic colorectal cancer. Oncotarget 7: 75013-75022, 2016.

41. Adams EF, Rafferty B, Mower J, Ward H, Petersen B and Fahlbusch R: Human acoustic neuromas secrete interleukin-6 in cell culture: Possible autocrine regulation of cell proliferation. Neurosurgery 35: 434-438, 1994

42. Taurone S, Bianchi E, Attanasio G, Di Gioia C, Ierinó R, Carubbi C, Galli D, Pastore FS, Giangaspero F, Filipo R, et al: Immunohistochemical profile of cytokines and growth factors expressed in vestibular schwannoma and in normal vestibular nerve tissue. Mol Med Rep 12: 737-745, 2015.
43. Ferraro A, Boni T and Pintzas A: EZH2 regulates cofilin activity and colon cancer cell migration by targeting ITGA 2 gene. PLoS One 9: e115276, 2014.

44. Yang Q, Bavi P, Wang JY and Roehrl MH: Immuno-proteomic discovery of tumor tissue autoantigens identifies olfactomedin 4 CD11b, and integrin alpha-2 as markers of colorectal cancer with liver metastases. J Proteomics 168: 53-65, 2017.

45. Dong J, Wang R, Ren G, Li X, Wang J, Sun Y, Liang J, Nie Y, Wu K, Feng B, et al: HMGA2-FOXL2 axis regulates metastases and epithelial-to-mesenchymal transition of chemoresistant gastric cancer. Clin Cancer Res 23: 3461-3473, 2017.

46. Ban EZ, Lye MS, Chong PP, Yap YY, Lim SYC and Abdul Rahman H: Haplotype CGC from XPD, hOGG1 and ITGA2 polymorphisms increases the risk of nasopharyngeal carcinoma in Malaysia. PLoS One 12: e0187200, 2017.

47. Liu X, Liang Z, Gao K, Li H, Zhao G, Wang S and Fang J: MicroRNA-128 inhibits EMT of human osteosarcoma cells by directly targeting integrin $\alpha 2$. Tumour Biol 37: 7951-7957, 2016.

48. Ding W, Fan XL, Xu X, Huang JZ, Xu SH, Geng Q, Li R, Chen D and Yan GR: Epigenetic silencing of ITGA2 by MiR-373 promotes cell migration in breast cancer. PLoS One 10: e0135128, 2015.

49. Ma HY, Liu XZ and Liang CM: Inflammatory microenvironment contributes to epithelial-mesenchymal transition in gastric cancer. World J Gastroenterol 22: 6619-6628, 2016.

50. McNab F, Mayer-Barber K, Sher A, Wack A and O'Garra A: Type I interferons in infectious disease. Nat Rev Immunol 15: 87-103, 2015.

51. Wang Y, Li P, Wang B, Wang S and Liu P: Identification of myeloid-derived suppressor cells that have an immunosuppressive function in NF2 patients. J Cancer Res Clin Oncol 145: 523-533, 2019.

52. Bonetti B, Valdo P, Ossi G, De Toni L, Masotto B, Marconi S, Rizzuto N, Nardelli E and Moretto G: T-cell cytotoxicity of human Schwann cells: TNFalpha promotes fasL-mediated apoptosis and IFN gamma perforin-mediated lysis. Glia 43: 141-148, 2003.

53. Cao L, Yang G, Gao S, Jing C, Montgomery RR, Yin Y, Wang P, Fikrig $\mathrm{E}$ and You F: HIPK2 is necessary for type I interferonmediated antiviral immunity. Sci Signal 12: eaau4604, 2019. 